\title{
GASTROSCHISIS ASSOCIATED WITH COLONIC ATRESIA - CASE REPORT
}

\author{
Ivona Đorđevic $^{1,2}$, Anđelka Slavkovic ${ }^{1,2}$, Zoran Marjanovic ${ }^{1,2}$, Dragoljub Živanovic $^{1,2}$, Milan Petrovic $^{2 *}$ \\ ${ }^{1}$ Pediatric Surgery Clinic, Clinical Center Niš, Serbia \\ ${ }^{2}$ University of Niš, Faculty of Medicine, Niš, Serbia
}

\begin{abstract}
We present an extremely rare case of gastroschisis associated with colonic atresia, anomaly seen in only 1.5$5 \%$ of all gastroschisis patients. Surgery for this anomaly can be very difficult due to the concomitant inflammation or other anomalies (mesenterium communae, volvulus, etc). Although prolonged intestinal dismotility is expected in these patients, mechanical obstruction must not be excluded in postoperative course, as it is explained in this case. Our case report demonstrates that primary colonic anastomosis is safe and well tolerated surgical procedure for these patients and that obstructive ileus is possible days after primary surgery and must not be overlooked.
\end{abstract}

Key words: gastroschisis, colonic atresia, treatment.

\section{Introduction}

Gastroschisis (laparoshisis) is a rare congenital defect, which is characterized by abdominal organ prolapse through the small defect of the abdominal wall, usually localized to the right from the umbilicus. Untill today the exact cause and pathogenesis of gastroschisis remain unclear. Prolapsed organs can be small intestine, colon, liver, spleen, stomach, sometimes the ovaries. In most cases gasroschisis is an isolated defect [1] About 5-22\% of infants born with gastroschisis will have associated anomalies of the digestive tract, such as atresia or stenosis of the small intestines or colon, malrotation, etc. [2].

\section{Case report}

A 37-week-old term male newborn, with prenatally diagnosed gastroschisis was born via uncomplicated vaginal delivery, with body weight of 3150 grams and APGAR scores 9/10. Abdominal wall defect to the right from umbilicus was detected with narrow aperture od 2 $\mathrm{cm}$ in diameter and with prolapsed abdominal organs (mostly small and large bowel) (Figure 1).

After initial oropharyngeal aspiration and reanimation, all prolapsed intestines were carefully wrapped in sterile warmed gauze, to prevent iatrogenic volvulus, and the patient was transfered to our neonatal intensive care unit (NICU) for further treatment. After standard preopeative preparation, blood tests, abdominal ultra-

*Correspondence to: Ivona Đorđević, M.D., PhD

Faculty of Medicine, University of Niš

81 Dr. Zoran Djindjić Blvd, 18000 Niš, Serbia

Phone: +281631096800

E-mail: ivonadj74@gmail.com

Received February $17^{\text {th }}, 2017$, accepted for publication October $23^{\text {th }}, 2017$

${ }^{*} \mathrm{PhD}$ Student sound and abdomen and chest radiography, the patient was considered an appropriate candidate for reduction of gastroschisis and primary closure of abdominal wall. Nasogastric (NG) tube was placed for bowel decompression, and there was no meconium after gentle rectal irrigation. Furthermore, by "milking" of dilated bowel loops, the obstruction caused by transverse colon atresia was noticed (Figure 2), and solved by two-layers endoblique anastomosis of the colon, establishing the intestinal continuity (Figure 3).

The remaining prolapsed abdominal organs were reduced into the abdominal cavity without increasing intraabdominal pressure. The abdominal defect was primarily closed without complications and the patient was transferred to the NICU. Antibiotic therapy and total parenteral nutrition (TPN) were administered. Postoperatively, daily rectal irrigation started on day 7 in order to activate bowel motility, but without signs of spontaneous meconium evacuation. NG tube even 14 days after surgery evacuated abundant gastric residuum. Ultrasound exami-

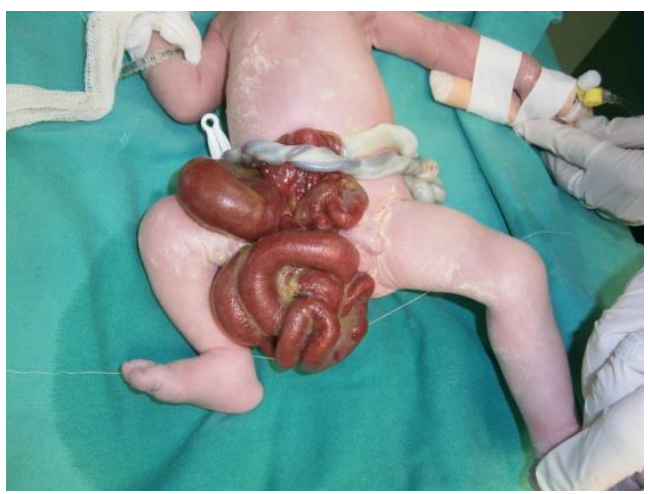

Fig. 1 Patient with gastroschisis. Eviscerated organs are mainly small bowel and colon. 


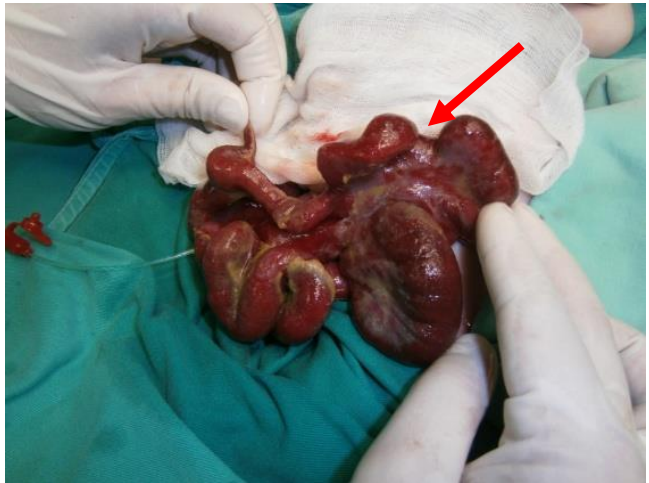

Fig. 2 Atretic colon at in the level of colon transversum (arrows), about $30 \mathrm{~cm}$ from appendix vermiformis (between surgeon's fingers)

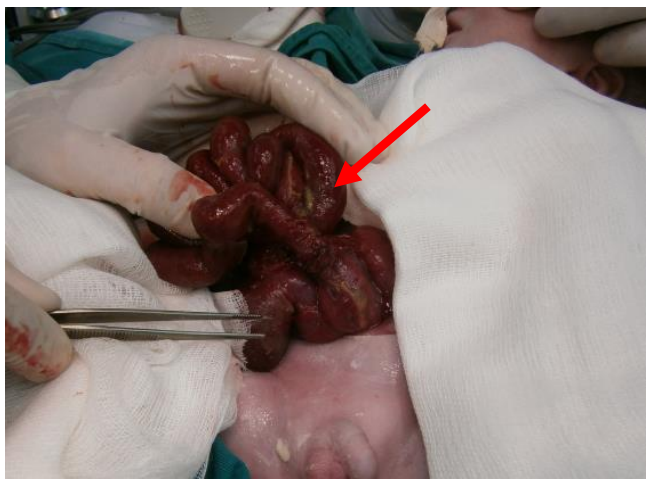

Fig. 3 Primary end-oblique colonic anastomosis (arrow)

nation described extremely dilated aperistaltic bowel loops with lots of free fluid in the abdominal cavity, and air-fluid levels on X ray. Relaparotomy was done fifteen days after primary surgical repair of gastroschisis, discovering "kinking" of colonic loop just few centimeters proximal to the anastomosis site, blocking intestinal passage. Intestinal passage was reestablished immediately after adhesiolysis and the anastomosis was intact. The patient was again transferred to NICU. Further postoperative course was uneventful and the patient was discharged from hospital after 32 days. He has been regularly monitored since then. The boy is now 4 years old with no complications so far.

\section{Discussion}

Gastroschisis is congenital ventral body wall defect with increasing prevalence and incidence of 1 per 2000 live births [3]. Isolated colonic atresia represents a very uncommon entity and the rarest cause of neonatal intestinal obstruction with the incidence of 1 per 20.000 live births and it comprises about $1.8-5 \%$ of intestinal atresias. Furthermore, the simultaneous occurrence of these two entities is extremely rare. Diagnosis of atresia is often difficult at the initial exploration of the prolapsed intestines because of inflammatory coating covering the bowel, resulting in overlooked associated intestinal atresia in patients with gastroschisis [4].
The pathogenesis of gastroschisis still remains controversial. Various theories and hypotheses regarding this defect are discussed in the literature [5,6], but vascular disruption theory is the most common. According to this theory, gastroschisis is a result of premature involution of right umbilical vein leading to ischemia and forming the weak spot (mesenchymal defect) at the junction of body stalk and future abdominal wall [7]

Considering the extremely rare association of two entities (gastroschisis and colonic atresia) there are no larger series to describe precisely the pathogenesis. However, vascular insult or strangulation of eviscerated intestines in pinched abdominal ring with intestinal infarction strongly support the vascular hypotheses. Intestinal atresia is a consequence of ischemic injury caused by extrinsic mesenteric vascular obstruction in narrow abdominal ring or in utero volvulus [8].

Although intestinal atresias can be diagnosed synchronously with gastroschisis on antenatal ultrasound, in most cases atresias are diagnosed postnatally, even after primary abdominal wall closure, due to the early feeding intolerance during recovery. Contrast radiographic studies can mark the atretic portions of intestines.

Generally, the most important consideration for the surgeon is to find the most adequate surgical approach for treating this small group of patients. Restoring normal bowel function remains the focus of care, and largely depends on the assessment of reactive alterations of the intestine. Intestinal atresia significantly increases morbidity and mortality, so surgeons are still debating about the best method for treating infants with gastroschisis and concomitant atresias. There is no perfect and unique method, and treatment of colonic atresia can be quite challenging. Kronfly published studies suggesting that bowel resection with primary anastomosis is a reasonable treatment for infants with gastroschisis and associated colonic atresia proximal to the splenic flexure, and a colostomy with delayed anastomosis for colonic atresia distal to the splenic flexure [9].

Naturally, surgeons should not insist on primary anastomosis and rational surgical assessment is essential. Intestinal repair at the first operation is sometimes possible and depends on the severity of the inflammatory coating. Unfortunately, this scenario is rarely feasible. If patients are unable to undergo primary anastomosis, restoration of bowel function must be delayed. In this circumstance, the combination of primary abdominal wall closure and enterostomy is the most appropriate strategy. Stoma closure should be planned for second stage, after few weeks, depending on the child's condition. When patients with such anomalies are managed appropriately, outcomes are generally favorable.

In our case we delayed the second operation because we felt that prolonged ileus was the result of gastroschisis itself, which was obviously an incorrect assumption. It is well described in literature that incidence of adhesive ileus and mechanical obstruction is very high, up to $30 \%$ [10-12], so delayed intestinal dysmotility should not be understood as a usual complica- 
tion in the postoperative course, but should raise suspicion on possible mechanical obstruction.

\section{Conclusion}

The management of gastroschisis associated with intestinal atresias has improved in recent decades mainly due

\section{References}

1. Benjamin B, Wilson GN. Anomalies associated with gastroschisis and omphalocele: Analysis of 2825 cases from the Texas Birth Defects Registry. J of Paediatr Surg 2014; 49:514-519.

2. Akhtar J, Skarsgard ED. Associated malformations and the "hidden mortality" of gastroschisis. J Pediatr Surg 2012; 47:911-916

3. Eggink BH, Richardson CJ, Malloy MH, Angel CA. Outcome of gastroschisis: a 20-year case review of infants with gastroschisis born in Galveston, Texas. J Pediatr Surg 2006; 41(6):1103-1108

4. Bauman Z, Nanagas V Jr. The Combination of Gastroschisis, Jejunal Atresia, and Colonic Atresia in a Newborn. Case Report in Pediatr2015; 6:1-4.

5. De Vries PA. The pathogenesis of gastroschisis and omphalocele. J Pediatr Surg1980; 15:245-251.

6. Hoyme HE, Higginbottom MC, Jones KL. The vascular pathogenesis of gastroschisis: intrauterine interruption of the omphalomesenteric artery. J Pediatr 1981; 98(2):228-231. to improved care in NICU, TPN and plenty of different individualized surgical approaches available, regarding the intestinal status. Although prolonged intestinal dysmotility is expected in these patients, mechanical obstruction must not be excluded in postoperative course.

7. Van Allen MI, Smith DW. Vascular pathogenesis of gastroschisis. J Pediatr 1981; 98(4):662-663.

8. Etensel B, Temir G, Karkiner A. Atresia of the colon. J Pediatr Surg 2005; 40:1258-1268

9. Kronfli R, Bradnock TJ, Sabharwal A. Intestinal atresia in association with gastroschisis: a 26-year review. Ped Surg Int 2010; 26(9):891-894.

10. Di Lorenzo C, Youssef NN. Diagnosis and management of intestinal motility disorders. Semin Pediatr Surg 2010; 19(1):50-58.

11. Phillips JD, Raval MV, Redden C, Weiner TM. Gastroschisis, atresia, dysmotility: surgical treatment strategies for a distinct clinical entity. J Pediatr Surg 2008; 43(12):2208-2212.

12. Lund $\mathrm{CH}$, Bauer $\mathrm{K}$, Berrios $\mathrm{M}$. Gastroschisis: incidence, complications, and clinical management in the neonatal intensive care unit. J Perinat Neonatal Nurs 2007; 21(1):63-68. 